\title{
Variable Lung Density Consideration in Attenuation Correction of Whole-Body PET/MRI
}

\author{
Harry R. Marshall ${ }^{1,2}$, Frank S. Prato ${ }^{1-3}$, Lela Deans ${ }^{1}$, Jean Théberge ${ }^{1-3}$, R. Terry Thompson ${ }^{1-3}$, and Robert Z. Stodilka ${ }^{1-4}$ \\ ${ }^{1}$ The Lawson Health Research Institute, Imaging Program, London, Ontario, Canada; ${ }^{2}$ Department of Medical Biophysics, \\ The University of Western Ontario, London, Ontario, Canada; ${ }^{3}$ Department of Diagnostic Imaging, St. Joseph's Health Care, London, \\ Ontario, Canada; and ${ }^{4}$ London Health Sciences Centre, London, Ontario, Canada
}

Present attenuation-correction algorithms in whole-body PET/ MRI do not consider variations in lung density, either within or between patients; this may adversely affect accurate quantification. In this work, a technique to incorporate patient-specific lung density information into MRI-based attenuation maps is developed and compared with an approach that assumes uniform lung density. Methods: Five beagles were scanned with ${ }^{18} \mathrm{~F}-\mathrm{FDG}$ PET/CT and MRI. The relationship between MRI and CT signal in the lungs was established, allowing the prediction of attenuation coefficients from MRI. MR images were segmented into air, lung, and soft tissue and converted into attenuation maps, some with constant lung density and some with patient-specific lung densities. The resulting PET images were compared by both global metrics of quantitative fidelity (accuracy, precision, and root mean squared error) and locally with relative error in volumes of interest. Results: A linear relationship was established between MRI and CT signal in the lungs. Constant lung density attenuation maps did not perform as well as patient-specific lung density attenuation maps, regardless of what constant density was chosen. In particular, when attenuation maps with patient-specific lung density were used, precision, accuracy, and root mean square error improved in lung tissue. In volumes of interest placed in the lungs, relative error was significantly reduced from a minimum of $12 \%$ to less than $5 \%$. The benefit extended to tissues adjacent to the lungs but became less important as distance from the lungs increased. Conclusion: A means of using MRI to infer patient-specific attenuation coefficients in the lungs was developed and applied to augment whole-body MRI-based attenuation maps. This technique has been shown to improve the quantitative fidelity of PET images in the lungs and nearby tissues, compared with an approach that assumes uniform lung density.

Key Words: PET/MRI; attenuation correction; lung density; segmentation; whole-body imaging

J Nucl Med 2012; 53:977-984

DOI: 10.2967/jnumed.111.098350

\footnotetext{
Received Sep. 15, 2011; revision accepted Feb. 21, 2012.

For correspondence or reprints contact: Harry Marshall, Lawson Health Research Institute, Imaging Department, 268 Grosvenor St., London, ON, Canada, N6A 4V2.

E-mail: hmarshall@lawsonimaging.ca

Published online May 7, 2012.

COPYRIGHT (c) 2012 by the Society of Nuclear Medicine, Inc.
}

A fter a decade and a half of development, human whole-body PET/MRI systems are now a reality (1). It has been widely speculated that PET/MRI will prove useful in several clinical disciplines (2-4), a prediction that is in the nascent stages of realization $(5,6)$. However, without a means of attenuation correction (AC), accurate quantification in PET is not possible.

Multiple approaches have been proposed to create MRIbased attenuation maps ( $\mu$-maps) (7-15). However, none of these approaches measures the attenuation coefficients ( $\mu$-coefficients) of the lungs, which vary both between individuals (16) and within a given individual $(17,18)$ and are influenced by inflation $(16,17,19)$, gravitational dependency $(18,19)$, and pathology $(18,20,21)$.

Visualizing lung parenchyma with MRI is challenging. The lungs have a low proton density (22) and short transverse relaxation time $\left(\mathrm{T}_{2} *\right)(23,24)$, compromising available MRI signal. Also, the lungs are mobile and highly vascular, generating motion and flow artifacts, respectively (22).

In this work, an MRI-based AC method that incorporates patient-specific measures of lung $\mu$-coefficients is developed. First, a standard MRI pulse sequence capable of visualizing lung tissue is described. Next, the relationship between MRI signal and CT signal in the lungs is inferred. Said relationship was used to create $\mu$-maps with patientspecific $\mu$-coefficients. The quantitative fidelity of PET reconstructions induced by these $\mu$-maps was compared with reconstructions done with $\mu$-maps that assumed a constant $\mu$-coefficient in the lungs across all subjects.

\section{MATERIALS AND METHODS}

\section{Experimental Protocol}

Five canines were scanned with PET/CT and MRI. One set of PET emission data was collected per canine, but CT scans were acquired at 3 respiratory states to simulate different lung densities. The CT scans yielded a clinical quality $\mathrm{CT}\left(\mathrm{CT}_{\mathrm{clin}}\right)$ and a pre$\mu$-map for $\mathrm{AC}\left(\mathrm{CT}_{\text {pre- } \mu}\right)$. Two types of $\mathrm{MR}$ image were acquired: one of the whole body (MRI $\mathrm{WB}_{\mathrm{WB}}$ ) and one of the lungs ( $\left.\mathrm{MRI}_{\text {lung }}\right)$. As with CT scans, MR images were acquired at 3 respiratory states. Further, MRI $_{\text {lung }}$ was acquired at 4 echo times (TEs), enabling the computation of a lung $\mathrm{T}_{2} *$ map $\left(\mathrm{MRI}_{\text {lungT2* }}\right)$ and extrapolated proton density image $\left(\mathrm{MRI}_{\text {lungPD }}\right)$. The $\mathrm{MRI}_{\text {lung }}$ image 
with the shortest TE was given the name $\mathrm{MRI}_{\text {lungSTE}}$, where STE stands for short TE.

The MRI signal was related to $\mathrm{CT}_{\text {clin }}$ signal in the lungs rather than 511-keV $\mu$-maps because the latter exhibited severe partialvolume effects. First, $\mathrm{MRI}_{\text {lungste }}$ was registered to $\mathrm{CT}_{\text {clin }}$. Three scatterplots of $\mathrm{CT}_{\text {clin }}$ lung signal versus $\mathrm{MRI}_{\text {lungSTE }}$ signal were produced: one relating signal intensities at individual voxels, one relating mean signal intensities of coronal slices-coronal slices were chosen to preserve the dorsal to ventral lung density gradient that arises in supine subjects $(17,18)$-and the last relating mean signal intensities of the lungs in their entirety. Linear regression was performed on each scatterplot, and the resulting mappings were termed voxel-by-voxel, slice-by-slice, and global, respectively. The process was repeated for $\mathrm{MRI}_{\text {lungT2* }}$ and $\mathrm{MRI}_{\text {lungPD, }}$ but for reasons discussed in the "Results" section, only MRI lungSTE was used to create $\mu$-maps.

Next, multiple MRI-derived pre- $\mu$-maps (CT-like objects that the PET/CT scanner converts to $\mu$-maps) were formed. First, $\mathrm{MRI}_{\mathrm{WB}}$ was segmented into air, lung, and soft tissue. Air and soft tissue were assigned values of $-1,000$ and 0 Hounsfield units (HU), respectively. Three pre- $\mu$-maps were formed by registering $\mathrm{MRI}_{\text {lungSTE }}$ to $\mathrm{MRI}_{\mathrm{WB}}$ and applying the voxel-by-voxel, slice-byslice, or global mappings to the lungs. PET reconstructions using these pre- $\mu$-maps are denoted $\mathrm{PET}_{\text {voxels }}, \mathrm{PET}_{\text {slices }}$, and $\mathrm{PET}_{\text {global }}$, respectively. Eleven pre- $\mu$-maps were formed by assigning the lungs a constant $\mathrm{CT}$ number ranging from -900 to $-400 \mathrm{HU}$ in $50-\mathrm{HU}$ increments. PET reconstructions using these pre- $\mu$-maps are referred to by subscripting the CT number assigned to the lungs, for example, PET -650 . Reconstructions using any MRIor CT-based $\mu$-map are termed $\mathrm{PET}_{\mathrm{MRI}}$ and $\mathrm{PET}_{\mathrm{CT}}$, respectively.

The quality of the $\mathrm{PET}_{\mathrm{MRI}}$ reconstructions was assessed by comparison to $\mathrm{PET}_{\mathrm{CT}}$. The analysis included both global and local components. Statistical testing was done with ANOVA and Tukey tests.

\section{Subjects}

This work was conducted on 5 female beagles (mass, $8-12 \mathrm{~kg}$ ). The protocol was approved by The University of Western Ontario's animal care committee.

Anesthesia was initiated with propofol and maintained with $2.0 \%-2.5 \%$ isoflurane. After intubation, artificial ventilation was conducted with a Veterinary ADS 1000 system (Engler Engineering Co.). To facilitate coregistration of the PET/CT and MR images, the canines were immobilized on a rigid board during the experiment.

\section{Imaging}

Imaging consisted of ${ }^{18} \mathrm{~F}$-FDG PET/CT performed on a Discovery VCT (GE Healthcare) and MRI on a Verio 3-T (Siemens Medical).

After an overnight fast (mimicking the clinical protocol for whole-body PET/CT), the ${ }^{18} \mathrm{~F}-\mathrm{FDG}$ was administered intravenously $1 \mathrm{~h}$ before the PET/CT study. The injected activity was approximately $10 \mathrm{MBq} / \mathrm{kg}$. The acquisition was 3-dimensional, with 5 min per table stop. Reconstructions were done with ordered-subset expectation maximization (2 iterations, 28 subsets). The PET images had an in-plane pixel size of $5.47 \times 5.47 \mathrm{~mm}$ $(128 \times 128$ matrix size $)$, with $3.27-\mathrm{mm}$ slice thickness. The acquisition was ungated.

CT scans were acquired at 3 respiratory states: functional residual capacity (FRC) was attained by halting ventilation, and 2 levels of inspiration were achieved by applying a positive inspiratory pressure (PIP) of $8 \mathrm{~cm}$ of $\mathrm{H}_{2} \mathrm{O}$ and $16 \mathrm{~cm}$ of $\mathrm{H}_{2} \mathrm{O}$.
All CT scans were $140 \mathrm{kVp}$ and $110 \mathrm{mAs}$ and were reconstructed with filtered backprojection. $\mathrm{CT}_{\text {clin }}$ had an in-plane pixel size of $0.98 \times 0.98 \mathrm{~mm}$, matrix size of $512 \times 512$, and slice thickness of $3.27 \mathrm{~mm}$. $\mathrm{CT}_{\text {pre- } \mu}$ had a larger in-plane pixel size $(1.37 \times 1.37 \mathrm{~mm})$. The scanner converted $\mathrm{CT}_{\text {pre }-\mu}$ into a $\mu$-map by downsampling via voxel averaging to a $128 \times 128$ matrix size, smoothing with a gaussian filter (full width at half maximum $\approx 10$ $\mathrm{mm}$ ), and then applying a lookup table.

The MRI protocol was repeated for each respiratory state. $\mathrm{MRI}_{\mathrm{WB}}$ was a 2-dimensional turbo fast low-angle shot (TurboFLASH), with a repetition time of $392 \mathrm{~ms}$, TE of $1,300 \mu \mathrm{s}, 1$ signal average, in-plane pixel size of $1.95 \times 1.95 \mathrm{~mm}$, matrix size of $128 \times$ 128 , axial slice thickness of $5 \mathrm{~mm}$ without gaps, flip angle of $10^{\circ}$, pixel bandwidth of $630 \mathrm{~Hz}$ per pixel, and anterior or posterior phase encode direction. The acquisition was spread over 4 table positions aligned with the isocenter, each consisting of 36 slices acquired in $15 \mathrm{~s} . \mathrm{MRI}_{\text {lung }}$ was also acquired with Turbo-FLASH, but with some altered parameters: a repetition time of $121 \mathrm{~ms}$, TE of $750 \mu \mathrm{s}$, in-plane pixel size of $1.87 \times 1.87 \mathrm{~mm}$, coronal slice thickness of $10 \mathrm{~mm}$ without gaps, pixel bandwidth of $1,532 \mathrm{~Hz}$ per pixel, and right or left phase encode direction. The image was acquired in 1 table position and consisted of 18 slices. $\mathrm{MRI}_{\text {lung }}$ was cardiacgated in diastole via a 3-lead electrocardiogram. Imaging time was approximately $10 \mathrm{~s}$. MRI lung was repeated 3 more times with 3 different TEs of 850, 950, and 1,030 $\mu$ s to compute MRI lungT2* $_{\text {la }}$ via a voxel-by-voxel exponential fit as a function of TE. MRI lungSTE $_{\text {I }}$ was normalized to the signal from a reference vial of saline placed next to the canine's neck. MRI lungPD was computed by voxel-byvoxel extrapolation of $\mathrm{MRI}_{\text {lungSTE }}$ to TE of 0 via $\mathrm{MRI}_{\text {lungT2*. }}$

Radiofrequency transmission was via the body coil, and signal reception was through elements of the spine array, body matrix, and large flex radiofrequency coils adjacent to the field of view. The body matrix was secured loosely to avoid obstructing chest wall expansion. Prescan normalization was performed before each acquisition to reduce radiofrequency shading.

\section{Image Registration}

$\mathrm{MRI}_{\text {lungSTE}}, \mathrm{MRI}_{\text {lungT2*, }}$, and $\mathrm{MRI}_{\text {lungPD }}$ were registered to $\mathrm{CT}_{\text {clin }}$ to generate the mappings. Resolution was matched by voxel averaging. Registration was performed using the Insight Segmentation and Registration Toolkit (ITK) (25). Mattes mutual information was chosen as the similarity measure. Because, at FRC, there was excellent anatomic agreement between $\mathrm{CT}_{\text {clin }}$ and $\mathrm{MRI}_{\text {lung }}$, a rigid transform was used. For the other 2 respiratory states, the diaphragm's position oscillated slightly. To accommodate the minor discrepancies in lung inflation, a hierarchic transform model was chosen, evolving sequentially from rigid to affine to nonrigid (26).

Similarly, $\mathrm{MRI}_{\text {lungSTE }}$ was registered to $\mathrm{MRI}_{\mathrm{WB}}$, and completed MRI-based pre- $\mu$-maps were rigidly registered to the CT-based pre- $\mu$-maps. Once aligned, the background of the CT-based pre$\mu$-map (e.g., patient bed and rigid board securing the canines' positioning) was added to the MRI-based pre- $\mu$-map.

\section{Image Segmentation}

$\mathrm{MRI}_{\mathrm{WB}}$ was segmented into air, lung, and soft tissue using a semiautomated algorithm developed in-house and implemented on Matlab (version 7.9.0.529; The MathWorks). The details of the segmentation algorithm have been described previously (27). Briefly, the subject's body was identified using an empiric threshold. Voxels outside the body were deemed air. The lungs were 
segmented by applying a level-set algorithm implemented in ITK-SNAP (28) to seed voxels identified with a second empiric threshold. The air in the trachea was segmented by hand, and segmentation errors were corrected manually. All remaining voxels were classified as soft tissue.

\section{Quantitative Analysis}

The global quality of PET $_{\text {MRI }}$ images was assessed in lung, soft tissue, and bone. Masks of these tissues were generated by thresholding the CT-based pre- $\mu$-map $(-950 \mathrm{HU}<$ lung $\leq-150 \mathrm{HU}<$ soft tissue $\leq 100 \mathrm{HU}<$ bone). Only axial slices containing lung were included in the analysis.

For each PET $_{\text {MRI }}$ reconstruction, a voxel-by-voxel scatterplot of PET $_{\text {MRI }}$ activity versus PET $_{\text {CT }}$ activity was created in lung, soft tissue, and bone. The scatterplots were normalized to the maximum PET $_{\mathrm{CT}}$ activity. Three metrics were computed on each scatterplot: the integral from 0 to 1 of the squared difference between the line of best fit (LOBF) computed via linear regression and the line of identity, $D_{y=x}^{2}$; the squared Pearson product-moment correlation coefficient, $R^{2}$; and the root mean square error, $E$, defined as $\sqrt{\frac{1}{n} \sum_{i=1}^{n}\left(y_{i}-x_{i}\right)^{2}}$, where $y_{i}$ and $x_{i}$ are the (normalized) estimated and true activities, respectively, at voxel $i$, and $n$ is the number of voxels. $D_{y=x}^{2}$ measures the proximity of the line of best fit to the line of identity - that is, accuracy. $R^{2}$ measures the spread of points about the line of best fit-that is, precision. $E$ is affected by both accuracy and precision; in particular, $E$ approaches zero if and only if $D_{y=x}^{2}$ approaches 0 and $R^{2}$ approaches 1 .

Each metric was analyzed statistically using a 3-way AVOVA ( $\alpha=0.05$ ), with respiratory state, tissue type, and $\mu$-map as factors. Significant results were followed by the Tukey test.

Local magnitude of relative error $(\%)$ was assessed in eight $1.094 \times 1.094 \times 0.981 \mathrm{~cm}$ rectangular volumes of interest (VOIs) (Supplemental Fig. 1; supplemental materials are available online only at http://jnm.snmjournals.org). Statistical analysis was the same as above, except the tissue type factor was changed to VOI in the ANOVA.

Steps were also taken to identify experimental errors. Lung segmentation error in $\mathrm{MRI}_{\mathrm{WB}}$ was computed as the percentage difference in lung volume at FRC as compared with $\mathrm{CT}_{\text {clin. }}$. To see whether these errors were dependent on lung inflation, this computation was repeated for the other respiratory states, and the means and SD were compared statistically with a 1-way ANOVA and Bartlett test for equal variances, respectively.

The effect of misregistration between $\mathrm{MRI}_{\text {lung }}$ and $\mathrm{CT}_{\text {clin }}$ on the voxel-by-voxel mapping (most sensitive to registration error) was inferred by shifting the registered $\mathrm{MRI}_{\text {lung }}$ by -1 to $+1 \mathrm{~cm}$ in 0.5 -mm increments along each of the $x, y$, and $z$ axes and recalculating $R^{2}$ as a function of the shift.

\section{RESULTS}

Of $\mathrm{MRI}_{\text {lungsTe }}, \mathrm{MRI}_{\text {lungt2*}}$, and $\mathrm{MRI}_{\text {lungPD }}$, only $\mathrm{MRI}_{\text {lungSTE }}$ correlated with CT; thus, neither MRI $\mathrm{I}_{\text {lungT2* }}$ nor $\mathrm{MRI}_{\text {lungPD }}$ were included in the subsequent analysis (Supplemental Fig. 2). Good agreement between the spatial distribution of lung signal in CT and $\mathrm{MRI}_{\text {lungSTE }}$ can be observed in Supplemental Figure 3. The mappings from $\mathrm{MRI}_{\text {lungSTE }}$ to $\mathrm{CT}$ number are presented in Figure 1. A linear relationship was demonstrated between the modalities.
The MRI-based pre- $\mu$-maps that incorporate lung information are contrasted with a CT-based pre- $\mu$-map in Figure 2 . The global mapping retains the least spatial information, whereas the voxel-by-voxel mapping retains the most.

The results of the tissue-specific analysis are presented in Figure 3, and the ANOVA results are found in Table 1. The results of the post hoc test are best visualized in Figure 3; points without overlapping error bars are significantly different. The $\mu$-map class had little influence on the metrics in soft tissue and bone, but a marked impact in the lungs.
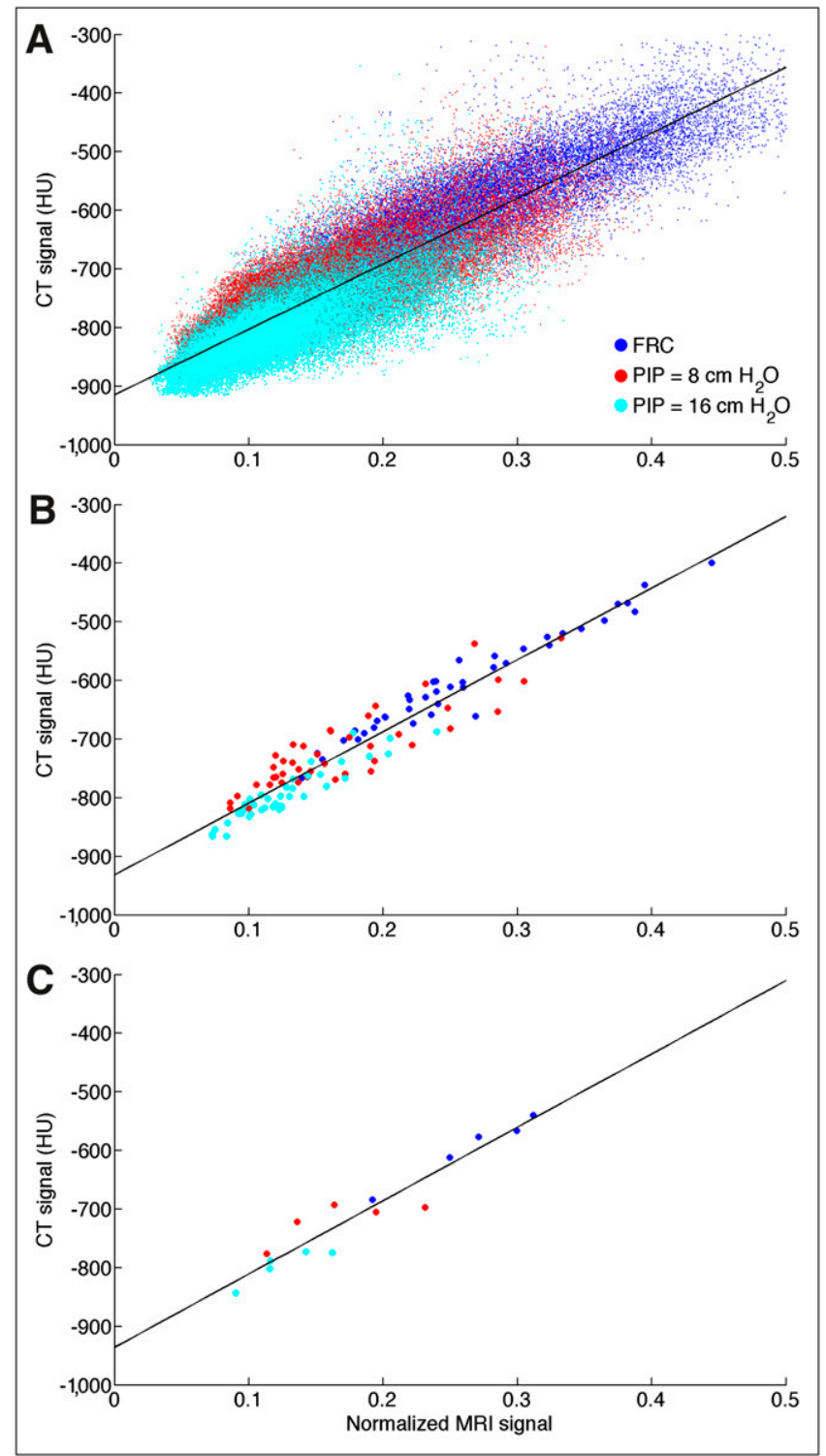

FIGURE 1. Relationship between MRI lungSTE signal and CT number. Points on scatterplots represent voxels $(A)$, coronal slices $(B)$, and all lung tissue $(C)$. Line-of-best-fit equations are $y=1,116 x-$ $916, y=1,223 x-932$, and $y=1,251 x-937$, respectively. Data from all 5 canines are plotted on each scatterplot. Each point's color represents canine's respiratory state, where dark blue is FRC, red is PIP $=8 \mathrm{~cm}$ of $\mathrm{H}_{2} \mathrm{O}$, and light blue is PIP $=16 \mathrm{~cm}$ of $\mathrm{H}_{2} \mathrm{O}$. As expected, increased volume of air in lungs reduces both MRI and CT signals. 

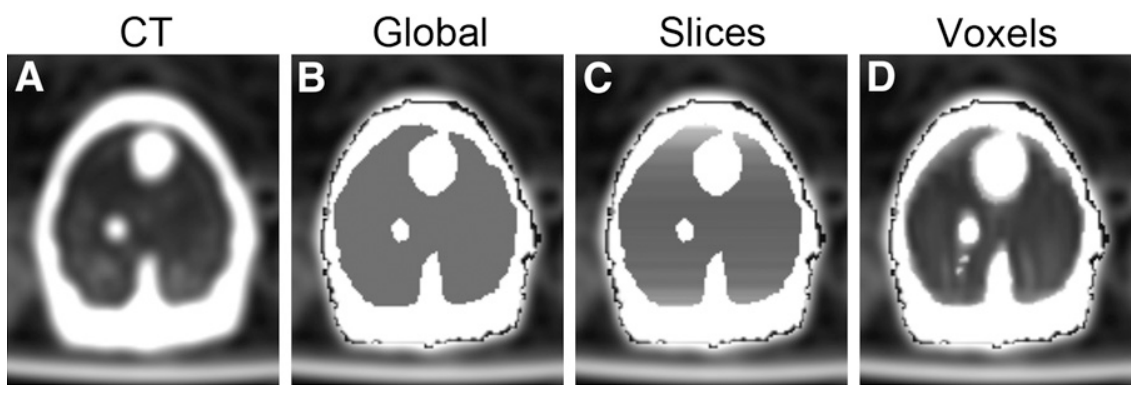

Within the lungs, $\mathrm{PET}_{\text {voxels }}$ exhibited the best accuracy, as reflected by $D_{y=x}^{2}$. $\mathrm{PET}_{\text {slices }}$ and $\mathrm{PET}_{\text {voxels }}$ were the most precise, as reflected by $R^{2}$. The lowest $E$ was achieved by PET $_{\text {voxels }}$, followed by PET $_{\text {slices }}$.

The VOI analysis (Table 2) revealed that the minimum error in the lungs, heart, and liver was achieved by $\mathrm{PET}_{\text {voxels, }}$, $\mathrm{PET}_{\text {global }}$, and $\mathrm{PET}_{\text {slices }}$, respectively. Error was minimized in the chest wall, vena cava, and vertebral bodies by PET -900 , $\mathrm{PET}_{-700}$, and $\mathrm{PET}_{-400}$, respectively. However, in many VOIs the differences were not statistically significant.

Representative profiles through the PET images are presented in Figure 4. The effect of altering the lungs' $\mu$-coefficients is propagated into nearby soft tissues, including the myocardium, diminishing as the distance from the lungs increases. In this example PET $_{\text {voxels }}$ is the most representative of the profile through $\mathrm{PET}_{\mathrm{CT}}$.

The lungs were undersegmented at FRC by $16 \% \pm 8 \%$, at $\mathrm{PIP}=8 \mathrm{~cm}$ of $\mathrm{H}_{2} \mathrm{O}$ by $14 \% \pm 12 \%$, and at $\mathrm{PIP}=16 \mathrm{~cm}$ of $\mathrm{H}_{2} \mathrm{O}$ by $18 \% \pm 11 \%$. Neither the means $(P=0.82)$ nor the SDs $(P=0.81)$ were significantly different between the respiratory states.

Regarding the effect of misregistration on the voxel-byvoxel mapping, $R^{2}$ was found to be an approximately gaussian function of shift, peaking at 0.8 when no shift was applied. To maintain $R^{2}$ within $10 \%$ of its optimum, the maximum allowable shift was $\pm 1.25 \mathrm{~mm}$ left to right and $\pm 2.75 \mathrm{~mm}$ anterior to posterior or superior to inferior.

\section{DISCUSSION}

In this work, a means of using MRI to infer the spatial $\mu$-coefficient distribution in the lungs was developed and tested on 5 canines. The evidence suggests doing so improves quantification in PET images.

In Figure 3, observe that in lung tissue the $\mu$-map class influences each of the 3 metrics of quantitative fidelity; this was statistically significant in all cases (Table 1). Moreover, PET $_{\text {voxels }}$ performed the best according to all 3 metrics. Additionally, $\mathrm{PET}_{\text {voxels }}$ had the least error in the lung VOIs (Table 2). These results suggest that $\mathrm{PET}_{\text {voxels }}$ is the best choice for quantification in the lung.

Though $\mu$-map choice did not influence quantification in soft tissue or bone when averaged over the whole thorax (Fig. 3), it did affect structures near the lungs (Fig. 4), notably the vena cava and peripheral left ventricle (Table 2). In the latter, there was a benefit to estimating the lung's

$\mu$-coefficients, which is potentially of clinical significance because even subtle alterations in viability or perfusion PET can affect clinical impression (29). However, in the vena cava (which crudely simulated a pulmonary lesion), PET $_{-700}$ performed the best. This may have been due to the limitation of MRI-based AC algorithms relying on segmentation. In Figure 2A, the vena cava in the CT-based $\mu$-map was subject to the partial-volume effect. Segmentation cannot reproduce this phenomenon because each voxel must be classified as air, lung, or soft tissue. In the MRI-based $\mu$-maps (Figs. 2B-2D), the voxels about the vena cava were preferentially deemed soft tissue. The MRI-based estimates of activity were therefore inflated relative to $\mathrm{PET}_{\mathrm{CT}}$. One means of compensating is to underestimate the lung's $\mu$-coefficients. As the mean lung CT number across all subjects and respiratory states was $-600 \mathrm{HU}, \mathrm{PET}_{-700}$ 's apparent success was probably attributable to undervaluing the true mean by $100 \mathrm{HU}$. Further, PET voxels $_{\text {achieved the }}$ lowest SD in the vena cava, suggesting that albeit biased, it is the most precise and therefore amenable to correction with a scaling factor. In sum, accurate quantification of lung lesions in PET/MRI may prove challenging even with patient-specific estimates of the lungs' $\mu$-coefficients.

As the amount of adjacent lung tissue near the VOI decreases, so does the lung's impact on quantification. For instance, in the central left ventricle, vertebral body, liver's dome, and chest wall, the error was never changed by more than $9 \%$ on the basis of $\mu$-map selection, and no statistically significant differences emerged. In these regions, what $\mu$-coefficients are assigned to the lungs is therefore less important.

The importance of reliable quantification in the heart and lung lesions is clear, but several potential applications of PET/MRI depend on accurate PET images of the lung parenchyma itself. For instance, whereas PET has demonstrated utility in identifying lung inflammation and infection in cystic fibrosis (30), CT-based AC is undesirable considering the predominantly pediatric patient population and ionizing radiation that accompanies CT scans; MRIbased AC could provide a convenient alternative, consistent with the "Image Gently" campaign. PET/MRI may also prove useful in understanding the inflammatory response in acute lung injury; pulmonary models of ${ }^{18} \mathrm{~F}$-FDG kinetics (31) might be complemented by functional measures such as perfusion via MRI (32). There are several other 


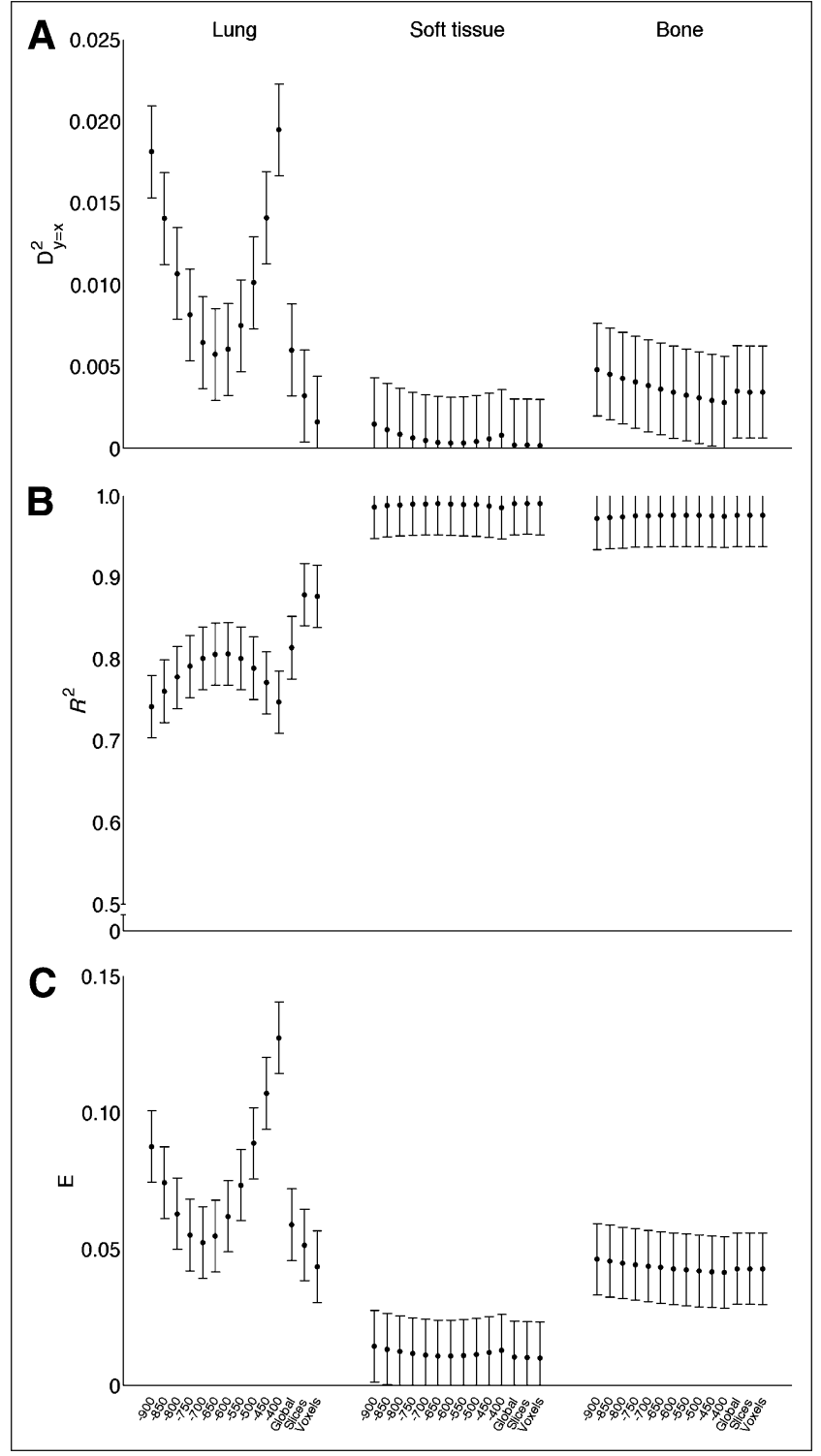

FIGURE 3. Three metrics of $\mathrm{PET}_{\mathrm{MRI}}$ 's quantitative fidelity broken down by tissue type and $\mu$-map class. Metrics include $D_{y=x}^{2}(\mathrm{~A}), R^{2}$ $(\mathrm{B})$, and $E(\mathrm{C})$. Leftmost blocks of data are from within lungs, middle blocks from soft tissue, and rightmost blocks from bone. Each point within given block corresponds to different $\mathrm{PET}_{\mathrm{MRI}}$ reconstruction. Error bars indicate $95 \%$ confidence intervals.

instances in which PET/MRI may be useful for lung imaging (33), all of which will require accurate MRI-based AC of the lungs.

To our knowledge, no other approach has been able to measure the lungs' $\mu$-coefficients. Some assign a constant $\mu$-coefficient to the lungs $(9,12,13,27)$ whereas others permit $\mu$-coefficient distributions (15), but none are patientspecific. One method allows patient-specific $\mu$-coefficients to be estimated by an iterative reconstruction algorithm (14), but correct convergence is not guaranteed. Ours is the first approach to directly link MRI to CT signal in the lungs but is subject to the following limitations.
TABLE 1

Results of ANOVAs on Canine PET $\mathrm{MRI}_{\text {I }}$ Metrics of Overall Quantitative Fidelity and Quantitation Error in Myocardium

\begin{tabular}{lcrc}
\hline \multicolumn{1}{c}{ Metric } & Factor & $\mathrm{F}$ & $P$ \\
\hline$D_{y=x}^{2}$ & Respiratory state & 4.45 & 0.01 \\
& Tissue & 271.41 & $<0.0001$ \\
$R^{2}$ & $\mu$-map class & 11.45 & $<0.0001$ \\
& Respiratory state & 13.72 & $<0.0001$ \\
& Tissue & 899.97 & $<0.0001$ \\
$E$ & $\mu$-map class & 3.10 & 0.0001 \\
& Respiratory state & 0.13 & 0.88 \\
Magnitude of & Tissue & 570.71 & $<0.0001$ \\
relative error & $\mu$-map class & 9.35 & $<0.0001$ \\
in VOls & Respiratory state & 44.65 & $<0.0001$ \\
& & & \\
& & & \\
& VOI & 109.21 & $<0.0001$ \\
\hline
\end{tabular}

First, there remains a great deal of variance in Figure 1A unexplained by the regression. One source is the dependence of the MRI signal on magnetic timing parameters (notably $\mathrm{T}_{2} *$ ), which are location- and subject-specific (24). Pure proton-density images would avoid this problem, but our measures of $\mathrm{T}_{2}{ }^{*}$ proved too noisy to allow reliable extrapolation of the signal at $\mathrm{TE}=0 \mu \mathrm{s}$. Eliminating $\mathrm{T}_{2}{ }^{*}$ dependence may prove even more important if disease is present, but in this work the impact of disease on the relationship between MRI and CT lung signal was not assessed. Exploring how disease affects the connection between MRI and CT signal in the lungs should be a priority for future work in this area, because ultimately, the method is intended for patients with diseases.

Another contributor to the unexplained variance in the scatterplots in Figure 1 is registration error. By exploring the impact of misregistration via translation on $R^{2}$, it was found that there exists a 1.25 - to 2.75 -mm buffer (depending on direction) before the correlation deteriorates beyond $10 \%$. It is difficult to quantify registration error, especially for nonrigid transformations; however, given the precautions taken to immobilize the dogs, the visual agreement between the registered images, and the misregistration buffer, registration error likely had a limited impact on the results.

B1 and receiver inhomogeneity also add to the scatterplots' variance by making lung MRI signal a function of spatial position. However, prescan normalization reduced this problem. Retrospectively, it was found that the prescan normalization was also sufficient to standardize the MRI signal across subjects, rendering the additional step of normalizing to the signal from a saline vial unnecessary. This, however, may depend on the manufacturer.

Apart from the assignment of erroneous $\mu$-coefficients in the lungs, a major contributor to the observed errors in the PET $_{\text {MRI }}$ images was missegmentation. The improper classification of air, lung, and soft tissue can alter quantification, even in remote regions (27). Indeed, our analysis 

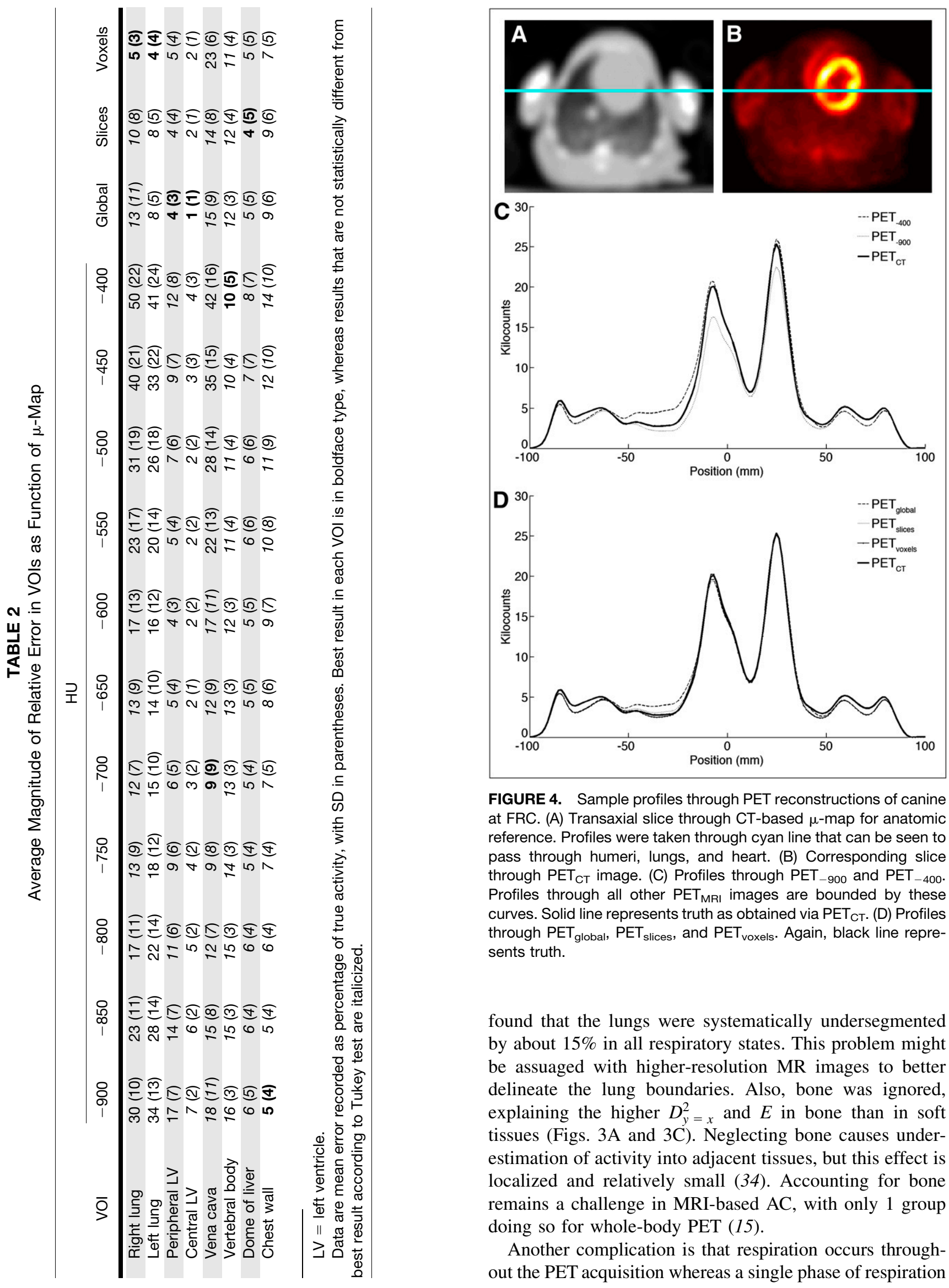

FIGURE 4. Sample profiles through PET reconstructions of canine at FRC. (A) Transaxial slice through CT-based $\mu$-map for anatomic reference. Profiles were taken through cyan line that can be seen to pass through humeri, lungs, and heart. (B) Corresponding slice through PET $_{\mathrm{CT}}$ image. (C) Profiles through PET -900 and PET -400 . Profiles through all other PET $_{M R I}$ images are bounded by these curves. Solid line represents truth as obtained via PET $C$. . (D) Profiles through $\mathrm{PET}_{\text {global }}, \mathrm{PET}_{\text {slices, }}$, and $\mathrm{PET}_{\text {voxels. }}$ Again, black line represents truth.

found that the lungs were systematically undersegmented by about $15 \%$ in all respiratory states. This problem might be assuaged with higher-resolution MR images to better delineate the lung boundaries. Also, bone was ignored, explaining the higher $D_{y=x}^{2}$ and $E$ in bone than in soft tissues (Figs. 3A and 3C). Neglecting bone causes underestimation of activity into adjacent tissues, but this effect is localized and relatively small (34). Accounting for bone remains a challenge in MRI-based AC, with only 1 group doing so for whole-body PET (15).

Another complication is that respiration occurs throughout the PET acquisition whereas a single phase of respiration 
was used to create the $\mu$-maps in this experiment, a problem referred to as transmission or emission mismatch. Thus, the $\mathrm{PET}_{\mathrm{CT}}$ image is not a true gold standard; it may contain errors of considerable magnitude (35). Nevertheless, these errors are separate from those induced by assigning erroneous $\mu$-coefficients to the lungs and do not alter the conclusions of this study. On the contrary, without a means to account for the changing lung $\mu$-coefficients with respiration $(16,17,19)$, optimal correction of the transmission to emission mismatch (via a time-varying $\mu$-map) would be flawed.

The principal reason that this study was performed using a large-animal model was that it allowed for precise control of their ventilation; the lungs could be held at the same respiratory state during the $\mathrm{CT}$ and MR image acquisitions. A means of controlling respiratory state in humans would be helpful to extend MRI-based estimates of lung $\mu$-coefficients to patients. An impediment to translating the methodology described here to humans is that people necessitate a larger field of view. Accordingly, if resolution were to be maintained, scan time and consequently breath-hold duration may increase. One solution is to eliminate cardiac gating, accelerating the acquisition. Gating was used in this study to facilitate image registration but was likely overconservative. However, eliminating cardiac gating is not a panacea. Additional problems will inevitably arise when modifying the pulse sequence for humans (e.g., altered signal-tonoise ratio and unanticipated artifacts), for which different solutions will be necessary. This method is not fully automated; if this approach is to be used clinically, automation is pivotal.

Most of the challenges associated with AC of the lungs in PET/MRI result from MRI's difficulty in reliably acquiring signal from lung parenchyma. An exciting prospect is to use ultrashort-TE pulse sequences to overcome lung tissue's short $\mathrm{T}_{2}{ }^{*}$. This method has been used to successfully demonstrate a correlation between signal intensity and lung inflation in mice (36). However, ultrashort-TE pulse sequences are harder to implement for large fields of view, and the acquisition generally takes several minutes. In the immediate future, standard gradient echo and turbo spin sequences with relatively short TEs are more tenable $(22,24)$.

\section{CONCLUSION}

Until now, MRI-based AC algorithms have treated the $\mu$-coefficients of the lungs as unknown, undermining quantification in PET images. We have demonstrated that MRI can be used to infer $\mu$-coefficients and applied this principle to MRI-based AC. As a result, quantification is clearly improved in the lungs and is likely improved in the surrounding tissues.

\section{DISCLOSURE STATEMENT}

The costs of publication of this article were defrayed in part by the payment of page charges. Therefore, and solely to indicate this fact, this article is hereby marked "advertisement" in accordance with 18 USC section 1734.

\section{ACKNOWLEDGMENTS}

Thanks to John Butler and John Patrick for their contributions to the MRI protocol development. This research was funded in part by a scholarship from the Natural Sciences and Engineering Research Council of Canada (NSERC) and grants from NSERC, the Canada Foundation for Innovation, the Canadian Institutes of Health Research, and the Ontario Research Fund. Further financial support was provided by Multi-Magnetics Incorporated. No other potential conflict of interest relevant to this article was reported.

\section{REFERENCES}

1. Herzog H, Pietrzyk U, Shah NJ, Ziemons K. The current state, challenges, and perspectives of MR-PET. Neuroimage. 2010;49:2072-2082.

2. Heiss W-D. The potential of PET/MR for brain imaging. Eur J Nucl Med Mol Imaging. 2009;36(suppl 1):S105-S112.

3. Sauter AW, Wehrl HF, Kolb A, Judenhofer MS, Pichler BJ. Combined PET/MRI: one step further in multimodality imaging. Trends Mol Med. 2010;16:508-515.

4. Nekolla SG, Martinez-Moeller A, Saraste A. PET and MRI in cardiac imaging: From validation studies to integrated applications. Eur J Nucl Med Mol Imaging. 2009;36(suppl 1):S121-S130.

5. Boss A, Bisdas S, Kolb A, et al. Hybrid PET/MRI of intracranial masses: initial experiences and comparison to PET/CT. J Nucl Med. 2010;51:1198-1205.

6. Uppal R, Catana C, Ay I, Benner T, Sorensen AG, Caravan P. Bimodal thrombus imaging: simultaneous PET/MR imaging with a fibrin-targeted dual PET/MR probe - feasibility study in rat model. Radiology. 2011;258:812-820.

7. Schreibmann E, Nye JA, Schuster DM, Martin DR, Votaw J, Fox T. MR-based attenuation correction for hybrid PET-MR brain imaging systems using deformable image registration. Med Phys. 2010;37:2101-2109.

8. Hofmann M, Steinke F, Scheel V, et al. MRI-based attenuation correction for PET/MRI: a novel approach combining pattern recognition and atlas registration. J Nucl Med. 2008;49:1875-1883.

9. Martinez-Möller A, Souvatzoglou M, Delso G, et al. Tissue classification as a potential approach for attenuation correction in whole-body PET/MRI: evaluation with PET/CT data. J Nucl Med. 2009;50:520-526.

10. Catana C, van der Kouwe A, Benner T, et al. Toward implementing an MRIbased PET attenuation-correction method for neurologic studies on the MR-PET brain prototype. J Nucl Med. 2010;51:1431-1438.

11. Keereman V, Fierens Y, Broux T, De Deene Y, Lonneux M, Vandenberghe S. MRI-based attenuation correction for PET/MRI using ultrashort echo time sequences. J Nucl Med. 2010;51:812-818.

12. Schulz V, Torres-Espallardo I, Renisch S, et al. Automatic, three-segment, MRbased attenuation correction for whole-body PET/MR data. Eur J Nucl Med Mol Imaging. 2011;38:138-152.

13. Steinberg J, Jia G, Sammet S, Zhang J, Hall N, Knopp MV. Three-region MRIbased whole-body attenuation correction for automated PET reconstruction. Nucl Med Biol. 2010;37:227-235.

14. Salomon A, Goedicke A, Schweizer B, Aach T, Schulz V. Simultaneous reconstruction of activity and attenuation for PET/MR. IEEE Trans Med Imaging. 2011;30:804-813.

15. Hofmann M, Bezrukov I, Mantlik F, et al. MRI-based attenuation correction for whole-body PET/MRI: quantitative evaluation of segmentation- and atlas-based methods. J Nucl Med. 2011;52:1392-1399.

16. Heremans A, Verschakelen L. Van fraeyenhoven L, Demedts M. Measurement of lung density by means of quantitative CT scanning. A study of correlations with pulmonary function tests. Chest. 1992;102:805-811.

17. Kalender WA, Rienmüller R, Seissler W, Behr J, Welke M, Fichte H. Measurement of pulmonary parenchymal attenuation: use of spirometric gating with quantitative CT. Radiology. 1990;175:265-268.

18. Gattinoni L, Pelosi P, Vitale G, Pesenti A, D'Andrea L, Mascheroni D. Body position changes redistribute lung computed-tomography density in patients with acute respiratory failure. Anesthesiology. 1991;74:15-23. 
19. Verschakelen JA. Van fraeyenhoven L, Laureys G, Demedts G, Baert AL. Differences in CT density between dependent and nondependent portions of the lung: influence of lung volume. AJR. 1993;161:713-717.

20. Hartley PG, Galvin JR, Hunninghake GW, et al. High-resolution CT-derived measures of lung density are valid indexes of interstitial lung disease. J Appl Physiol. 1994;76:271-277.

21. Kinsella M, Müller NL, Abboud RT, Morrison NJ. DyBuncio A. Quantitation of emphysema by computed tomography using a "density mask" program and correlation with pulmonary function tests. Chest. 1990;97:315-321.

22. Hatabu H, Chen Q, Stock KW, Gefter WB, Itoh H. Fast magnetic resonance imaging of the lung. Eur J Radiol. 1999;29:114-132.

23. Case TA, Durney CH, Ailion DC, Cutillo AG, Morris AH. A mathematical model of diamagnetic line broadening in lung tissue and similar heterogeneous systems: calculations and measurements. J Magn Reson. 1987;73:304-314.

24. Hatabu H, Alsop DC, Listerud J, Bonnet M, Gefter WB. T2* and proton density measurement of normal human lung parenchyma using submillisecond echo time gradient echo magnetic resonance imaging. Eur J Radiol. 1999;29:245-252.

25. Ibanez L, Schroeder W, Ng L, Cates J. The ITK Software Guide, 2nd ed. Clifton Park, NY: Kitware; 2005.

26. Rueckert D, Sonoda LI, Hayes C, Hill DLG, Leach MO, Hawkes DJ. Nonrigid registration using free-form deformations: application to breast MR images. IEEE Trans Med Imaging. 1999;18:712-721.

27. Marshall HR, Stodilka RZ, Theberge J, et al. A comparison of MR-based attenuation correction in PET versus SPECT. Phys Med Biol. 2011;56:4613-4629.
28. Yushkevich PA, Piven J, Hazlett HC, et al. User-guided 3D active contour segmentation of anatomical structures: significantly improved efficiency and reliability. Neuroimage. 2006;31:1116-1128.

29. Schelbert HR, Beanlands R, Bengel F, et al. PET myocardial perfusion and glucose metabolism imaging: Part 2 -Guidelines for interpretation and reporting. J Nucl Cardiol. 2003;10:557-571.

30. Klein M, Cohen-Cymberknoh M, Armoni S, et al. ${ }^{18}$ F-Fluorodeoxyglucose-PET/ CT imaging of lungs in patients with cystic fibrosis. Chest. 2009;136:1220-1228.

31. Schroeder T, Vidal-Melo MF, Musch G, Harris RS, Venegas JG, Winkler T. Modeling pulmonary kinetics of 2-deoxy-2-[ $\left.{ }^{18} \mathrm{~F}\right]$ fluoro-D-glucose during acute lung injury. Acad Radiol. 2008;15:763-775.

32. Hopkins SR, Prisk GK. Lung perfusion measured using magnetic resonance imaging: new tools for physiological insights into the pulmonary circulation. $J$ Magn Reson Imaging. 2010;32:1287-1301.

33. Chen DL, Kinahan PE. Multimodality molecular imaging of the lung. J Magn Reson Imaging. 2010;32:1409-1420.

34. Schleyer PJ, Schaeffter T, Marsden PK. The effect of inaccurate bone attenuation coefficient and segmentation on reconstructed PET images. Nucl Med Commun. 2010;31:708-716.

35. Erdi YE, Nehmeh SA, Pan T, et al. The CT motion quantitation of lung lesions and its impact on PET-measured SUVs. J Nucl Med. 2004;45:1287-1292.

36. Togao O, Tsuji R, Ohno Y, Dimitrov I, Takahashi M. Ultrashort echo time (UTE) MRI of the lung: assessment of tissue density in the lung parenchyma. Magn Reson Med. 2010;64:1491-1498. 